\title{
A CONCEPTUAL FRAMEWORK FOR AUTOMATIC AND DYNAMIC THESAURUS UPDATING
}

IN INFORMATION RETRIEVAL SYSTEMS

\author{
M.F. BRUANDEI
}

Laboratoire IMAG

B.P. 53X, 38041 GRENOBLE Cedex (France)

\section{ABSTRACT}

This paper aims at presenting a methodology for automatic thesaurus construction in order to help the search of documents and we want to obtain the development of classes for specific topics (for a given corpus) without a priori semantic information. Information contained in the thesaurus lead to new search formulations via automatic and/or user feedback. This presentation even being theoretical is oriented toward a database implementation.

\section{Preliminary remarks}

Different strategies used in Information Retrieval Systems must be developped to increase "recal1" and "precision" 8 ". The classic one is the construction of thesaurus. A thesaurus is usually defined as a set of terms (called descriptors) and a set of relations between these terms.

This study is made for an information retrieval system using an inverted file (bitmap, each keyword points to a set of documents containing this keyword). For formulating a request the user defines a set of keywords and boolean operators on this set (for example MISTRAL, GOLEMPASSAT, STAIRS systems). When entering a document into the database, a module (e.g. PIAF) ${ }^{4,5}$ generates stems from the data (several grammatical variants of the same word are reduced to a canonical form). We call this form an item.

\section{Thesaurus construction in the context}

$$
\text { of local documents }
$$

Our object is to find a method for the construction of non-hierarchical relations and the definition of item clusters from these relations. A point to be underlined is that this methodology could efficiently be used only on homogeneous collections of texts. To this purpose, we only consider a database subset : the local set of all documents returned from a given query. The local clustering method makes use of the common occurrences of items within a certain "neighborhood", this method has been studied by R. ATTAR and A.S. FRAENKEL (in "Local feedback in full-text retrieval") 1 .

Let be $D_{\ell}$ the local set of documents retrieved from a given query and $\mathrm{T}_{\ell}$ the set of items contained in $\mathrm{D}_{\ell}$. We define a metrical function which is inversely proportional to the distance between items in the same sentence. Each item is defined by its coordinates (DN, SN, IN) where $\mathrm{DN}$ is the document number, $\mathrm{SN}$ the sentence number and IN the item number within a sentence.
For any item $t \in T_{\ell}$, let $w_{t}(i)$ be the coordinate of the $i$ th occurrence of $t$.

For any couple $(s, t) \in T_{\ell} \times T_{\ell}$, we define $d=\left|w_{t}(i)-w_{S}(j)\right|$ the distance between the ith occurrence of $t$ and $j$ th occurrence of $s$.

In fact

$$
\text { (1) } d=\left|I N_{t}(i)-I N_{s}(j)\right| \text { with }\left\{\begin{array}{l}
D N_{t}(i)=D N_{s}(j) \\
\operatorname{SN}_{t}(i)=\operatorname{SN}_{s}(j)
\end{array}\right.
$$

Let be $F$ a function of the distance $d$ :

(2) $\quad F\left(w_{t}(i), w_{s}(j)\right)=\left\{\begin{array}{l}1 / d \text { if } w_{t}(i), w_{s}(j) \text { ar } \\ \text { in the same sentence } \\ \text { with } d \leq 20 \\ 0 \text { otherwise. }\end{array}\right.$

For $s$ and $t \in \mathrm{T}_{\ell}$ we define :

(3) $b(s, t)=\sum_{i j} \sum_{j} F\left(w_{t}(i), w_{s}(j)\right)$

where the summation is over all occurrences $i$ and $j$ of $s$ and $t$.

Remark : $b(s, t)=b(t, s)$.

In order to normalize the function, we take $\mu_{R}(s, t)=\frac{b(s, t)}{f(t)}$ where $f(t)$ is the number of occurrences of $t$ for all local documents $D_{\ell}$

$$
0 \leq \mu_{R}(s, t) \leq 1 .
$$

Through this function, we obtain for an item s a reference vector $R$ which is a list of items $t$ related to $s$, such as $\mu_{R}(s, t)$ is greater (or equal) than a threshold $\alpha$. These values form an eigen vector : $\mathrm{E}_{\mathrm{R}_{\mathrm{S}}}$.

\section{Taking into account new local information in thesaurus updating}

Without excluding for the thesaurus the search of hierarchical relations (specific or generic), we try to build a set or a group of items having a notion of "similarity" or "liaison" between themselves. This thesaurus is built as the answers of the used Information Retrieval System are analysed. It must be structured so that the updating should be dynamic and automatic; the implementation study has not yet been examined. The main problem of updating is to take into account "liaisons", "proximities" or "similarities" between the already registered items in the thesaurus and the new liaisons found after a new query.

For any query, we obtain a set of items related to $s$. Let be $R$ the previous reference vector ( $\mu_{R}$ its associated function) and $R_{S}^{\prime}$ the newly calculated vector ( $\mu_{K}$, its associated function). 
A new reference vector may be calculated from $R_{s}$ and $R_{s}^{\prime}$ using two functions $m(s, t)$ and $M(s, t)$ :

$$
m(s, t)=\frac{\operatorname{Min}\left(\mu_{R_{s}}(s, t), \mu_{R_{s}^{\prime}}(s, t)\right)}{1-\left|\mu_{R_{s}}(s, t)-\mu_{R_{s}^{\prime}}(s, t)\right|}
$$

$$
M(s, t)=\frac{\operatorname{Max}\left(\mu_{R_{s}}(s, t), \mu_{R_{s}^{\prime}}(s, t)\right)}{1+\left|\mu_{R_{s}}(s, t)-\mu_{R^{\prime}}(s, t)\right|}
$$

The function $M$ involves all the items $t$ which are related, or not, to $s$ in $R_{S}$ and $R^{\prime}$ (see Table I). The function $m$ allows us to consider only the items which are both in $R_{S}$ and in $R_{S}^{\prime}$ (see Table I) .

One might consider $\mathrm{m}$ and $\mathrm{M}$ to be respectively the union and intersection of items $t$ related to $s$.

\begin{tabular}{|c|c|c|c|}
\hline$\mu_{R_{s}}(s, t)$ & $\mu_{R_{s}^{\prime}}(s, t)$ & $\mathrm{m}=\frac{\operatorname{Min}\left(\mu_{R_{s}}, \mu_{R^{\prime}}\right)}{1-\left|\mu_{R_{s}}-\mu_{R_{s}^{\prime}}\right|}$ & $M=\frac{\left.\operatorname{Max}\left(\mu_{R_{s}}, \mu_{R^{\prime}}\right)_{s}\right)}{1+\left|\mu_{R_{s}}-\mu_{R_{s}^{\prime}}\right|}$ \\
\hline 0 & 1 & indeterminate & 0.5 \\
\hline 0 & 0.2 & 0 & 0.166 \\
\hline 0 & 0.8 & 0 & 0.44 \\
\hline 0.1 & 0.9 & 0.5 & 0.5 \\
\hline 0.1 & 0.8 & 0.33 & 0.47 \\
\hline 0.1 & 0.7 & 0.25 & 0.43 \\
\hline 0.1 & 0.6 & 0.2 & 0.40 \\
\hline 0.1 & 0.5 & 0.16 & 0.36 \\
\hline 0.1 & 0.4 & 0.142 & 0.30 \\
\hline 0.1 & 0.3 & 0.125 & 0.25 \\
\hline 0.1 & 0.2 & 0.11 & 0.18 \\
\hline 0.1 & 0.1 & 0.1 & 0.1 \\
\hline 0.5 & 0.5 & 0.5 & 0.5 \\
\hline$\overline{0 . \overline{9}}$ & 0.2 & 0.66 & 0.52 \\
\hline 0.9 & 0.4 & 0.8 & 0.6 \\
\hline 0.9 & 0.5 & 0.83 & 0.64 \\
\hline 0.9 & 0.6 & 0.85 & 0.69 \\
\hline 0.9 & 0.8 & 0.88 & 0.81 \\
\hline
\end{tabular}

Table I using the above functions $m$ and M (formulas (4), (5))

Functions $\mathrm{m}$ and $\mathrm{M}$ consider the weakest and the strongest bindings between items. Any association between $s$ and $t$ is meaningful only as regard to the "binding strength", that is to say the value of the association function.

Use of the functions $m$ and $M$ for thesaurus construction and updating

For an item $x$, only the items related to $\mathrm{x}$ in several local contexts must be considered in the thesaurus. Thus, it is necessary to keep records of the initial queries into a pseudo-thesaurus. In this pseudo-thesaurus is registered, for any item $x$, the set of items related to $x$ in one or more local contexts.

Let be

$$
P S_{x}=\left\{t / \mu_{P S}(x, t) \geq \alpha\right\}
$$

for $x$ belonging to the set of items $T,\left(T=U T_{\ell}\right)$. Concerning an item $x$ of $T_{\ell}$, three reference vectors (and their associated functions) can be yielded : $R_{x}, P S_{x}$ and $T$ which are the sets of items $t$ related to $x$ respectively considered in the treated local context, in one or. more local contexts kept in the pseudo-thesaurus, and in the global context kept in the thesaurus.
These sets can be void, also several cases can be encountered :

1) $\mathrm{PS}_{\mathrm{x}}$ and $\mathrm{T}_{\mathrm{x}}$ are not void

The updating process is performed in three steps :

Step 1 : Tréatment_of of_new_data

In order to know, if the newly calculated liaisons in $R_{x}$ already exist in other local context, we compare $R_{x}$ and $P S_{x}$.

only the common items of these two reference vectors are considered, and we form a temporary reference vector $\mathrm{P}_{\mathrm{x}}$ using the function $\mathrm{m}$ (formula (4)).

In $\mathrm{P}_{\mathrm{X}}$ only items from $\mathrm{R}_{\mathrm{x}}$ which are previously related to $x$ in at least one context are retained. The stronger connections are decreased (see Table 1) because we can suppose they are only local.

Step 2 : Thesaurus updating_procecedure The thesaurus updating is made in two different ways :

(i) if $\mathrm{P}_{\mathrm{x}}$ and $\mathrm{T}_{\mathrm{x}}$ contain the same items $\mathrm{t}$, on $1 \mathrm{y}$ the eigen vector $\mathrm{E}_{\mathrm{T}}$ (of $\mathrm{T}_{\mathrm{X}}$ ) is modified using the function $\mathrm{x}$ (formula (4)) ; 
(ii) if the items $t$ in $T_{x}$ are different from those occuring in $P_{x}$, then a new reference vector $T_{X}$ is constructed combinating the values of functions $\mu_{T_{X}}$ and $\mu_{P S}$ using $M$ (formula
(5)).

Remarks :

- We do not calculate the new association function between two items for $\mathrm{T}$ with $\mathrm{m}$ (formula (4)), because we do not introduce new items related to $x$ in the thesaurus, when new items appear in several local contexts.

- The function $M$ uses the common or not common items and introduces in the thesaurus the new items, which are related to $x$ in at least two local contexts.

Step 3 : Pseudo-thesaurus updating procedure The pseudo-thesaurus updating must take into account the new items occuring in $R x^{\text {. The new }}$ association function for $P S$ is calculated from the association function $\mu_{R}$ and the old association function $\mu_{\mathrm{PS}}$ using $M$ (formula (5)).

2) $\operatorname{PS}_{x}$ and $T_{x}$ are void

This case corresponds to the situation where $\mathrm{x}$ is never appeared in any local context. We create the reference vectors PS in the pseudo-thesaurus and $\mathrm{R}_{\mathrm{x}}$ with the association function $\mu_{\mathrm{R}_{\mathrm{x}}}\left(\mathrm{PS}_{\mathrm{x}}=\right.$ $R_{x}$ ). No information about $x$ is kept in the thesaurus $\left(T_{x}=\varnothing\right)$.

3) $\mathrm{PS}_{\mathrm{x}}$ is not void and $T_{x}$ is void

This corresponds to the case where $x$ is already appeared in only one local context. If $R_{x} \neq \varnothing$, then we can build the initial reference vector $\mathrm{T}$ in the thesaurus. We use the association function $m$ (formula (4)) calculated from the values of association functions $\mu_{R_{x}}$ and $\mu_{P S}$ (respectively contained in the eigen vectors ${ }_{R_{X}}$ and $E_{P S_{X}}$ ).

The present experimentation exhibits among the items related to $\mathrm{x}$ in $\mathrm{T}_{\mathrm{x}}$ (initial step) local synonyms, some global synonyms and many parasistic items. After a few thesaurus updatings the values of the association function for parasistic $i$ tems rapidly decrease, and the values for local and global synonyms increase. It is clear that reliability of such a thesaurus can be reached only after a large number of queries. In such a situation new updating procedures might be considered so that new parasistic items should not be introduced in $T_{X}$ (thus breaking the stability of $\left.\mathrm{T}_{\mathrm{x}}\right)$.

\section{Global treatment of thesaurus}

Let be $T$ the large set of items registered in the thesaurus. In order to classify $T$ (i.e. to split $T$ into classes of similar items), we consider the couple of reference vectors $\mathrm{T}_{\mathrm{x}}$ and $\mathrm{T}_{\mathrm{y}}$ ( so $\mathrm{E}_{\mathrm{T}_{\mathrm{x}}}$ and $\mathrm{E}_{\mathrm{T}}$ ) for any items $\mathrm{x}$ and $\mathrm{y}$.

Let be $r(x, y)$ a similarity measure :
(6) $r(x, y)=\frac{\sum_{T} \operatorname{Min}\left(\mu_{T}, \mu_{T}\right)}{\sum_{T} \operatorname{Max}\left(\mu_{T}, \mu_{T}\right)}$

(7) $d(x, y)=1-r(x, y)$ is a pseudo-distance whose range is $[0,1]$.

We can use an association matrix (i.e. term-term matrix) between items and found a partition of $T$ in equivalence classes. Moreover, this method hardly applies to a great many items and does not seem realistic for a large scale dictionnary ( 6000 or 10000 items, for example) which are common in information retrieval field. To overcome this drawback, we may try to build up the global association matrix from the local ones. Some ideas have been suggested ${ }^{2}$ using the fuzzy sets theory 6,13 but there are still theoretical approaches.

\section{Feedback query processing}

Number of papers are related to the feedback query processing $1,8,12$ and our approach is similar.

We think to adopt the following strategy, though we lack practical results to assert better "score" on queries.

After a query we have therefore a set $R_{s}$ of items related to $s$ (for each $s \in T_{\ell}$ ) and a partition of $\mathrm{T}_{\ell}$ into equivalence classes $\Gamma_{j}$. In the thesaurus we might have both a set $T$ ( $i$ tems related to $s$ ) and a partition of the global set $T$ into equivalence classes $\mathrm{C}_{i}$.

Several strategies can be used, they are detailed in an other paper 4 . We can use only local context, global context or both global and local context. We summarize some of the solutions below:

1) use of only global context A query is automatically generated with $t$ instead of $x$ when $t$ belongs to the reference vector $T_{x}$ and $\mu_{\mathrm{T}_{\mathrm{X}}}(\mathrm{x}, \mathrm{t})$ is greater or equal than a threshold If the user agrees, a new query is generated with $t$ when $x$ and $t$ are equivalent in the thesaurus.

2) use of both local and global context When an item $t$ is considered as "similar" to $x$ both in local context $\left(R_{x}\right)$ and in global context $\left(T_{x}\right)$ and $\mu_{R}(x, t) \leq \mu_{T}(x, t), t$ automatica11y replaces $x$ In the query. When $R_{x}$ and $T_{x}$ have common items, we can purpose to the user new queries with item $t$ appearing in $T_{x}$ but not in $\mathrm{R}_{\mathrm{x}}\left(\mu_{\mathrm{T}}(\mathrm{x}, \mathrm{t}) \geq \alpha\right)$.

As previously mentioned we can use the same strategy using the local equivalence classes $\Gamma_{j}$ and global equivalence classes $C_{i}$ (automatic feedback query processing with $x^{1} \in C_{i} \cap \Gamma_{j}$, and under user control with $x \in C_{i}$ but $x \notin C_{i} n^{j} \Gamma_{j}$ and $\left.C_{i} \cap \Gamma_{j} \neq \varnothing\right)$.

In this last case, we can think global synonymies allow to retrieve new documents originally left out. 
From the previous analysis, it seems that the best strategies should be those using both local and global contexts, but this needs to be verified.

\section{Conclusions}

We conclude from present experimentation on small number of french texts that the thesaurus updating method shall give horizontal thesaurus relations.

Moreover unexpected relation between items should appear in the thesaurus, that is association which strongly reflects the corpus' content and which could not a priori be established and enha nced.

The methodology presented above does not exclude any further intervention on the thesaurus to refine semantic information about some particular cases, such as modifying values of the association function for some items, enriching definition of synonyms.

Our next goal for such a design of the thesaurus is twofold :

1) we wish to make possible non boolean queries through the use of fuzzy keywords and subsequent improvement of dialogue ;

2) we wish to cluster documents with a dynamic indexing mechanism.

\section{REFERENCES}

1 R. ATTAR \& A.S. FRAENKEL

Local feedback in full text retrieval systems. Journal of ACM, vo1.20, $\mathrm{n}^{\circ} 3$, pp. 397-417, July 1977.

2 M.F. BRUANDET

A propos de la construction automatique d'un thesaurus flou dans un système de recherche d'information (système documentaire). Internal research report IMAG Grenoble, Juin 1980.

3 M.F. BRUANDET

A conceptual framework for automatic and dynamic thesaurus updating and for feedback query processing.

Processing of SECOND INTERNATIONAL CONFERENCE ON DATA BASES IN THE HUMANITIES AND SOCIAL SCIENCES, Madrid, Juin 1980.

4 J. COURTIN

Algorithmes pour le traitement interactif des langues naturelles.

Thèse d'Etat soutenue à 1'Universitê Scientifique et Médicale de Grenoble, INPG, Octobre 1977.
5 E. GRANDJEAN

Projet PIAF - Application à 1a documentation automatique : dêfinition et utilisation du produit prototype PIAFDOC.

Internal research report, IMAG Grenoble, 1979.

6 T. RADECKI

Mathematical model of information retrieval system based on the concept of fuzzy thesaurus.

Information processing and management, vol.12, pp. 313-318, Pergamon Press, 1976.

7 L. REISINGER

On fuzzy thesaurus.

COMPSTAT/4 - Proc. Symp. Computational statistics, Bruckman b, Fersh1 1, Schmetterer Vienna Physics Verlag.

8 G. SALTON

The smart retrieval system, experiments in automatic.

Document processing (ch.21 - the use of statistical significance in relevance feedback. J.S. Brown, P.D. Reilly), Prentice Hall, 1971 .

9 G. SALTON

Dynamic information processing.

Prentice Ha11 1975.

$10 \mathrm{G}$. SALTON and D. BERGMARK

Clustered file generation and its application to computer Science taxonomies. IF IP Information processing 77, pp. 441-447, North Holland publishing company.

$11 \mathrm{~W}$. SILVERT

Symmetric summation : a class of operations on fuzzy sets.

IEEE Trans. SMC, 1979.

12 C.T. YU, M.K. SIU

Effective automatic indexing using term addition and deletion. Journal of ACM, vol.12, $\mathrm{n}^{\circ} 2$, April 1978, pp. $210-225$.

13 L.A. ZADEH

Fuzzy sets, Information and control. pp. 338-353, 1965. 